Informes de la Construcción Vol. 61, 516, 53-55, octubre-diciembre 2009 ISSN: 0020-0883 elSSN: 1988-3234 doi: 10.3989/ic.09.008

\title{
Anclaje de barras en zapatas de hormigón
}

\section{Anchorage lenghts in concrete footings}

\author{
L. Calavera ${ }^{(*)}$
}

\section{RESUMEN}

Se analiza la regla simplificada de suponer, que para zapatas, el desarrollo de la longitud de anclaje se cuenta a $0,5 \mathrm{~h}$ a partir del extremo de las barras, para la tensión .que tenga la barra en ese punto.

Esta simplificación, está basada en la hipótesis de zapata rígida, pero el Eurocódico EC-2 no lo dice y la Instrucción EHE-08 la fija para zapatas flexibles.

Como para todas las presiones $\sigma_{t}$ de contacto con el suelo, la zapata más económica es la de menor canto posible, para suelos con $\sigma_{t} \leq 0,2$ $\mathrm{N} / \mathrm{mm}^{2}$ la solución correcta puede diferir notablemente de la regla establecida por ambas Normas.

458-7

Palabras Clave: estructuras de hormigón, cimentaciones, armaduras, anclaje, fisuración.

El anclaje de las barras de los emparrillados de armadura de zapatas aisladas presenta algunas particularidades:

-El anclaje de una barra, al estar las ortogonales del emparrillado en tracción, está en una situación más desfavorable que la normal.

- La presión del suelo sobre la zapata, $\sigma_{t^{\prime}}$ beneficia al anclaje, si bien para las presiones normales este efecto es muy débil.

-Como se indica en la Figura 1 (pág. siguiente), si la zapata realmente flecta, la cara inferior se alarga de $\mathrm{AB}$ a $\mathrm{A}^{\prime} \mathrm{B}^{\prime}$, pero a este alargamiento se oponen las tensiones de rozamiento $\mu \sigma_{\mathrm{t}}$. En una primera estimación, $\mu$ suele oscilar de 0,3 a 0,6 para
SUMMARY

The paper analyzes the simplified rule for footing whereby the anchorage length is developed from a distance equal to $0,5 \mathrm{~h}$, measured form the end of the footing.

This simplification is based on the assumption that the footings involved are stiff, but Eurocode EC-2 fails to mention that fact, while Spanish code EHE-08 prescribe the rule for flexible footing.

The value of the soil contact pressure $\sigma_{t}$ notwithstanding, the shallower a footing is, the more economical. Consequently, for soils with $\sigma_{t} \leq 0,2 \mathrm{~N} /$ $\mathrm{mm}^{2}$ the right solution could differs from the rules laid down in the above two standards.

Keywords: structures of concrete, foundations, steel reinforcement, anchorage, cracking.

suelos granulares y limosos y la adherencia puede en estos casos considerarse nula.

Un estudio detallado puede encontrarse en mi libro citado en la referencia (1). La conclusión es que la cara inferior no se alarga o se alarga considerablemente menos que lo que establece el cálculo habitual y por lo tanto las armaduras no están en tracción o están en valores moderados de tracción y no necesitan anclaje, o éste se reduce mucho respecto a los valores teóricos ${ }^{(1)}$.

- A esto debe añadirse que el hormigón de limpieza (hoy frecuentemente de la misma resistencia que el de la zapata), se incorpora realmente al del recubrimiento de las armaduras, constituyendo recubrimientos reales de 8 a $13 \mathrm{~cm}$, que, teóricamente,

(*) Dr. Ingeniero de Caminos, Canales y Puertos. INTEMAC, Madrid (España)

Persona de contacto/Corresponding author: jcalavera@intemac.es

Fecha de recepción: 09-03-09 Fecha de aceptación: 13-10-09 


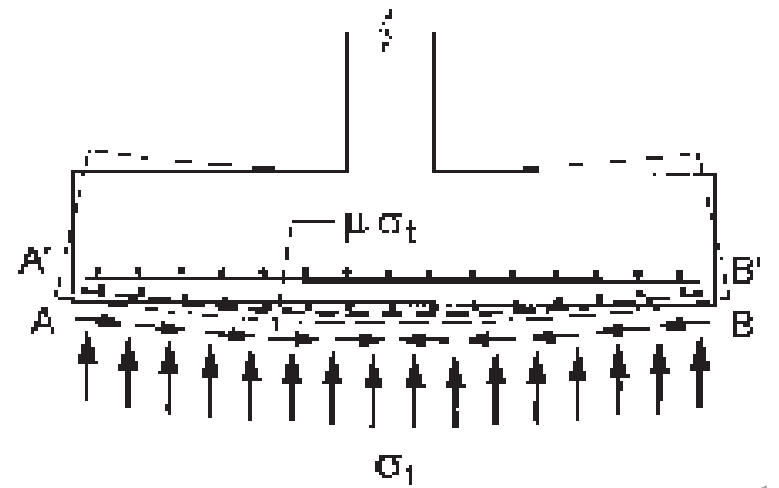

$\sigma_{1}$

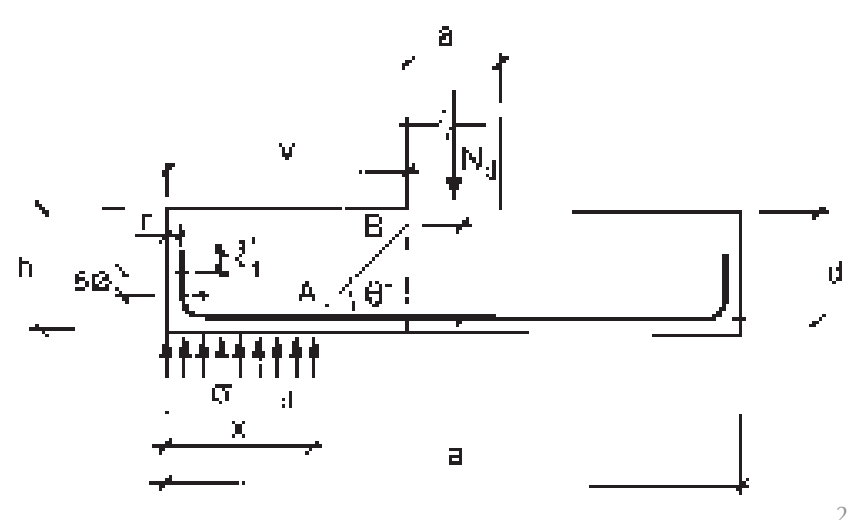

1. Conjunto de tensiones actuantes sobre la zapata.

2. Fuerzas de anclaje en función del ángulo $\theta$ de fisura de corte.
(2) El equilibrio se plantea para la faja de cimiento correspondiente a una barra. debían producir una fisuración amplia. No conocemos ningún caso de patología referente a este motivo.

Estos temas por el momento no se han considerado, que sepamos, en ninguna Norma. En el resto de este artículo no consideramos los tres aspectos particulares antedichos.

Consideremos la zapata indicada en la Figura 2 , donde $v \geq h$, es decir, que el cálculo de la zapata puede realizarse como flexible.

Es posible la formación de una fisura de corte con ángulo $\theta^{\circ}$. Como las cuantías de armadura son muy bajas, puede aceptarse $d=$ 0,9 h y la altura del punto de iniciación de la fisura, B, puede estimarse como 0,9 d, es decir $0,81 \mathrm{~h}$.

En lo que sigue a continuación aceptamos como simplificación el tomar momentos en la cara del pilar y no retranqueada e $=0,15$ a (esta simplificación es adoptada por muchas Normas).

La fuerza a anclar mediante la armadura, es la correspondiente al punto A, intersección del plano de la fisura con el eje de la armadura. Llamando $x$ a la longitud de $A$ al extremo de la zapata y tomando momentos en B.

$\mathrm{F}_{\mathrm{sd}, \mathrm{x}} \cdot 0,81 \mathrm{~h}=\mathrm{x} \sigma_{\mathrm{td}}\left(\mathrm{v}-\frac{\mathrm{x}}{2}\right)^{(2)}$

donde $\mathrm{F}_{\mathrm{sd}, \mathrm{x}}$ es el valor de cálculo de la fuerza de la barra de la armadura en $A ; \sigma_{t d}$ presión de cálculo sobre el suelo

Como $x=v-0,81 h \operatorname{cotg} \theta$

sustituyendo [2] en [1]

$\mathrm{F}_{\mathrm{sd}, \mathrm{x}} \cdot 0,81 \mathrm{~h}=\frac{\sigma_{\mathrm{td}}}{2}\left(v^{2}-0,656\right) \mathrm{h}^{2} \operatorname{cotg}^{2} \theta$ [3]

$F_{\text {sd, } x}=\sigma_{t d} \frac{v^{2}-0,656 h^{2} \operatorname{cotg}^{2} \theta}{1,62 h}$

Por otra parte, del momento flector aplicado se deduce

$A_{s} f_{y d} \cdot 0,81 h=\sigma_{t d} \cdot \frac{v^{2}}{2}$
$A_{s} f_{y d}=\frac{\sigma_{t d} \cdot v^{2}}{1,62 h}$

y por tanto la longitud de anclaje neta necesaria debe cumplir

$\frac{\mathrm{I}_{\mathrm{b}, \text { net }}}{\mathrm{I}_{\mathrm{b}}}=\frac{\mathrm{F}_{\mathrm{sd}, \mathrm{x}}}{\mathrm{A}_{\mathrm{s}} \mathrm{f}_{\mathrm{yd}}}$

donde $I_{b}$ corresponde a la posición de adherencia I, dada la posición de las barras.

Por tanto

$\mathrm{I}_{\mathrm{b}, \text { net }}\left[1-0,65\left(\frac{\mathrm{h}}{\mathrm{v}}\right)^{2} \operatorname{cotg}^{2} \theta\right] \mathrm{I}_{\mathrm{b}}$

De [2]

$x=v-0,81 h \operatorname{cotg} \theta$

Por lo tanto el menor valor de x, corresponde al máximo valor de $\operatorname{cotg} \theta$

Aquí se presenta una discordancia entre la Instrucción EHE-08 (2), que especifica en general $0,5 \leq \operatorname{cotg} \theta \leq 2$ y el Eurocódigo EC- 2 (3) que especifica $1 \leq \operatorname{cotg} \theta \leq 2,5$.

De acuerdo con EHE-08, el máximo valor de $\operatorname{cotg} \theta$ es 2 y por tanto:

$x=v-1,62 h=h\left(\frac{v}{h}-1,62\right)$

La función [8] se representa en la Figura 3.

Como se ve la regla de EHE-08, de disponer la longitud de anclaje a partir de una distancia igual a $0,5 \mathrm{~h}$, a partir del extremo del vuelo con la tensión de la barra correspondiente a ese punto, no es estrictamente válida más que para $v / h=2,12$.

De acuerdo con el Eurocódigo EC-2, el valor máximo de $\operatorname{cotg} \theta$ es 2,5 y por tanto

$\mathrm{x}=\mathrm{v}-2,025 \mathrm{~h} ; \quad \frac{\mathrm{x}}{\mathrm{h}}=\frac{\mathrm{v}}{\mathrm{h}}-2,025$

En la Figura 4 se representa la condición [9]. La regla de disponer la longitud de anclaje igual a $0,5 \mathrm{~h}$ a partir del extremo de la barra, sólo es estrictamente válida para $v / h=2,53$. 


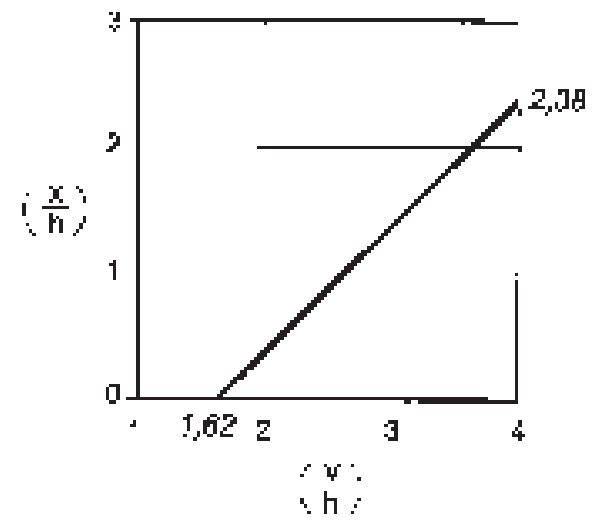

\section{CONCLUSIONES}

- La regla simplificada viene de la práctica europea de utilizar siempre zapatas con un vuelo $\underline{\mathrm{v}}<2 \mathrm{~h}^{(3)}$.

- Como en todo tipo de países, salvo los claramente subdesarrollados, los precios del hormigón y el acero conduce a que para cualquier presión admisible, la zapata más económica es la de canto mínimo, para tensiones bajas $\sigma_{t}$ sobre el terreno $\left(\sigma_{t}\right.$ $<0,20 \mathrm{~N} / \mathrm{mm}^{2}$ ) el cálculo conduce a valores de $v / h$ claramente superiores a los límites analizados anteriormente, siempre que sean compatibles con la hipótesis de reparto uniforme de presiones $\sigma_{\mathrm{td}}$.

- Para relaciones $v / h$ superiores a las indicadas, es conveniente calcular el valor de $x$

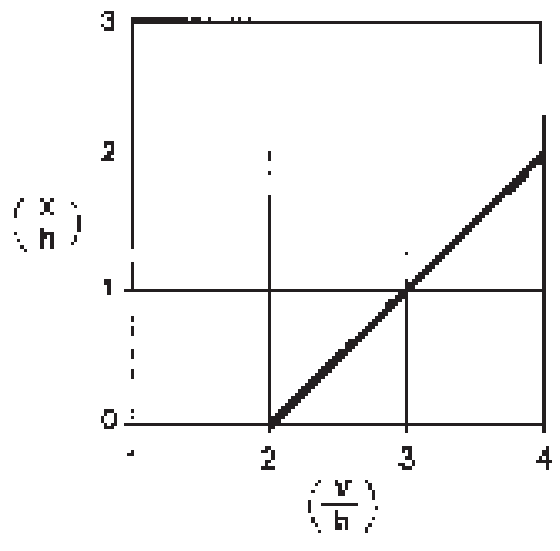

mediante la fórmula [8] y disponer el anclaje de acuerdo con la fórmula [7]. No hacerlo así, puede conducir a que si se emplean los diámetros más gruesos posibles, (como es lógico para abaratar el coste de la armadura), la fórmula [7], para valores de $\mathrm{v} / \mathrm{h}$ superiores a 2 , conduzca a un anclaje por prolongación recta mientras que la regla simplificada de suponer $\mathrm{x}=0,5 \mathrm{~h}$ conduzca, innecesariamente, a disponer patilla o eventualmente una prolongación recta vertical.

- La regla simplificada (que realmente simplifica un cálculo muy sencillo) puede conducir, en ciertos casos, a un encarecimiento de la ferralla por pasar de barras rectas, (lo que entra dentro de la terminología de "corte a medida"), a "barras conformadas". En este artículo no se analiza su nivel de seguridad.

\section{BIBLIOGRAFÍA}

(1) Calavera, J. "Cálculo de estructuras de cimentación". 4a Edición. INTEMAC. Madrid. 2000.

(2) EHE-08. "Instrucción para el proyecto y la ejecución de obras de hormigón estructural". M M de Fomento. Madrid 2008.

(3) EUROCÓDIGO EC-2. "Proyecto de estructuras de hormigón - Parte 1.1: Reglas generales y reglas para edificación". EUROPEAN COMMITTEE FOR STANDARDIZATION (CEN). Bruselas. 2004.

(4) EUROCÓDICO EC-2. "Proyecto de estructuras de hormigón - Parte 3: Cimentaciones de hormigón". EUROPEAN COMMITTEE FOR STANDARDIZATION (CEN). Bruselas. 1998. 